\title{
Nanoscale
}

CrossMark

$\leftarrow$ click for updates

Cite this: Nanoscale, 2016, 8, 10113

\section{Solution-based intramolecular singlet fission in cross-conjugated pentacene dimers $\uparrow$}

\author{
Johannes Zirzlmeier, ${ }^{a}$ Rubén Casillas, ${ }^{a}$ S. Rajagopala Reddy, ${ }^{\mathrm{b}}$ Pedro B. Coto, ${ }^{\mathrm{b}}$ \\ Dan Lehnherr, ${ }^{\mathrm{c}}$ Erin T. Chernick, ${ }^{\mathrm{d}}$ Ilias Papadopoulos, ${ }^{a}$ Michael Thoss, ${ }^{b}$ \\ Rik R. Tykwinski*d and Dirk M. Guldi*a
}

We show unambiguous and compelling evidence by means of pump-probe experiments, which are complemented by calculations using ab initio multireference perturbation theory, for intramolecular singlet fission (SF) within two synthetically tailored pentacene dimers with cross-conjugation, namely XC1 and XC2. The two pentacene dimers differ in terms of electronic interactions as evidenced by perturbation of the ground state absorption spectra stemming from stronger through-bond contributions in XC1 as confirmed by theory. Multiwavelength analysis, on one hand, and global analysis, on the other hand, confirm that the rapid singlet excited state decay and triplet excited state growth relate to SF. SF rate constants and quantum yields increase with solvent polarity. For example, XC2 reveals triplet quantum yields and rate constants as high as $162 \pm 10 \%$ and $(0.7 \pm 0.1) \times 10^{12} \mathrm{~s}^{-1}$, respectively, in room temperature solutions.

Received 25th March 2016, Accepted 29th March 2016

DOI: $10.1039 /$ c6nr02493a

www.rsc.org/nanoscale solution. ${ }^{25-28}$ In the solid state, tetracene features nearly degenerate singlet excited states and correlated pairs of triplet excited states, i.e., $E\left(\mathrm{~S}_{1}\right) \approx 2 E\left(\mathrm{~T}_{1}\right)$, rendering the standard enthalpy for intermolecular SF negligible. In contrast, the singlet excited state energy in pristine pentacene in the solid state is more than twice the energy of a correlated pair of two triplet excited states, namely $E\left(\mathrm{~S}_{1}\right) \geq 2\left(E\left(\mathrm{~T}_{1}\right)\right)$. Thus, $\mathrm{SF}$ is exoergic and unidirectional for pentacene. In hexacene, SF is linked to the formation of a correlated pair of triplet excited states and phonons. ${ }^{5,9,23,29-31}$

In contrast to SF in the solid state, SF in solution has only recently been discovered for pentacenes, with yields reaching as high as $200 \%{ }^{26-28}$ Solution-state SF has also been demonstrated in tetracene dimers, but with a SF yield of only $3 \%{ }^{25}$

It is logical to hypothesize that the relative proximity between neighboring acenes is critical to the process of SF, and the molecular geometry as well as the $\pi-\pi$ overlap would be dominant factors for efficient $\mathrm{SF}^{32-35}$ One can envision a plethora of acenes that might be synthesized to explore specific aspects of $\mathrm{SF}$ in dimeric structures. ${ }^{36-39}$ We have recently described the photophysical behavior of a set of three regioisomeric pentacene dimers, linked via an ortho-, meta-, and para-phenylene (Fig. 1). ${ }^{27}$ The observed triplet quantum yield in these dimers is as high as $156 \pm 5 \%$. It is interesting to note that the $\boldsymbol{m e t} \boldsymbol{a}$-linked dimer gives the best SF performance, a molecule in which the two pentacenes are not directly conjugated through the intervening phenylene ring, in contrast to either the ortho- or para-linked dimer. In light of the general structure of the meta-dimer, we speculate that moderating the electronic communication between two acene chromophores 

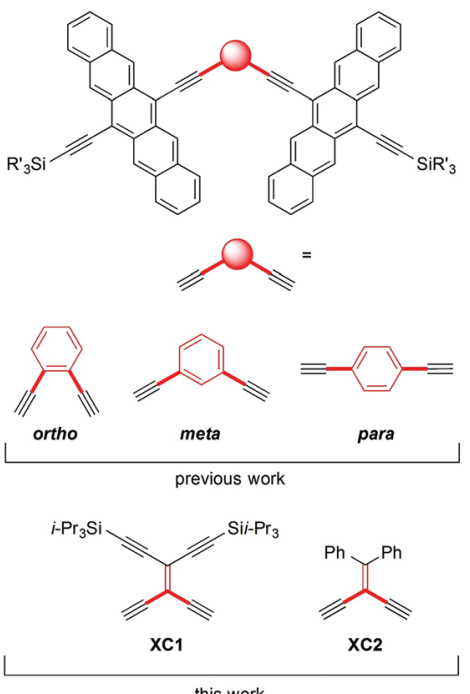

this work

Fig. 1 Phenylene tethered pentacene dimers studied previously ${ }^{27}$ and cross-conjugated pentacene dimers investigated in the present work.

might be an important design concept for the optimization of SF. In order to test this hypothesis, dimers based on diethynyland tetraethynylethenes (DEE and TEE, respectively) have been designed, in which the two pentacenes communicate in the ground state only via cross-conjugation. ${ }^{40,41}$ The constrained conjugation of DEEs and TEEs has been used, for example, to form molecular wires, ${ }^{42-44}$ switches, ${ }^{45}$ and nonlinear optical materials. ${ }^{46}$ Furthermore, the contrasting behavior of chromophores linked by meta-phenylene groups versus a cross-conjugated skeleton has been a topic of somewhat controversial discussion in organic electronics. ${ }^{47,48}$

Considering the excited states that are involved, their nature, and their electronic coupling, two main mechanisms for SF have been proposed over the years, the direct and the mediated, and the predominance of one or another remains a subject of controversy. ${ }^{2,7,9,17,32,49-62}$ In the direct mechanism, the absorbing state transforms into a correlated pair of triplet excited states, also called multiexcitonic (ME) or doubly excited state, which later dissociates into two (non-interacting) $\mathrm{T}_{1}$ states. In the mediated mechanism, the transformation of the absorbing state into the ME state, which then dissociates into two $T_{1}$ states, is facilitated by coupling to higher-lying CT states. $^{2,55}$ Recent studies have refined these traditional viewpoints, providing more information about the detailed interplay of the different electronic states involved in the process. In particular, one of the studies postulates that the initial excitation of the molecular system produces a state that is not an eigenstate of the system but a coherent superposition of the (lowest-lying) absorbing state, CT states, and the ME state., ${ }^{9,17}$ The latter ultimately splits into two separated $T_{1}$ states after decoherence. Finally, a very recent model suggests that the non-interacting $\mathrm{T}_{1}$ states are directly populated from the absorbing state without the participation of any intermediate electronic state in the process. ${ }^{62}$
In the present contribution, we have designed and examined two novel pentacene dimers XC1 and XC2, that feature a cross-conjugated framework to mediate the electronic coupling between the two pentacene moieties. The cross-conjugated structural modification is expected to be particularly helpful as a means to either enhance or attenuate the through bond electronic couplings between the pentacene chromophores. ${ }^{41}$ Furthermore, XC1 and XC2 feature rigid structures, with geometries that are similar to that found in ortho-dimer (vide infra).

\section{Results and discussion}

\section{Synthesis}

Cross-conjugated dimers XC1 and XC2 were synthesized from the reaction of masked pentacene $\mathbf{1}$, which has proven to be a valuable precursor for the stepwise assembly of pentacene derivatives via transition metal catalyzed reactions. ${ }^{27,37,39}$ Thus, the reaction of 1 with either dibromoolefin $2^{63}$ or $3^{64}$ was carried out using a Sonogashira cross-coupling protocol, and the TEE and DEE cores of $\mathbf{4}$ and $\mathbf{5}$ were formed in acceptable yields of $63 \%$ and $60 \%$, respectively (Scheme 1).

Subsequently, $\mathrm{Sn}$ (II)-mediated reductive aromatization afforded XC1 and XC2 in $82 \%$ and 95\% yield, respectively. Dimers XC1 and XC2 are stable to air and moisture under
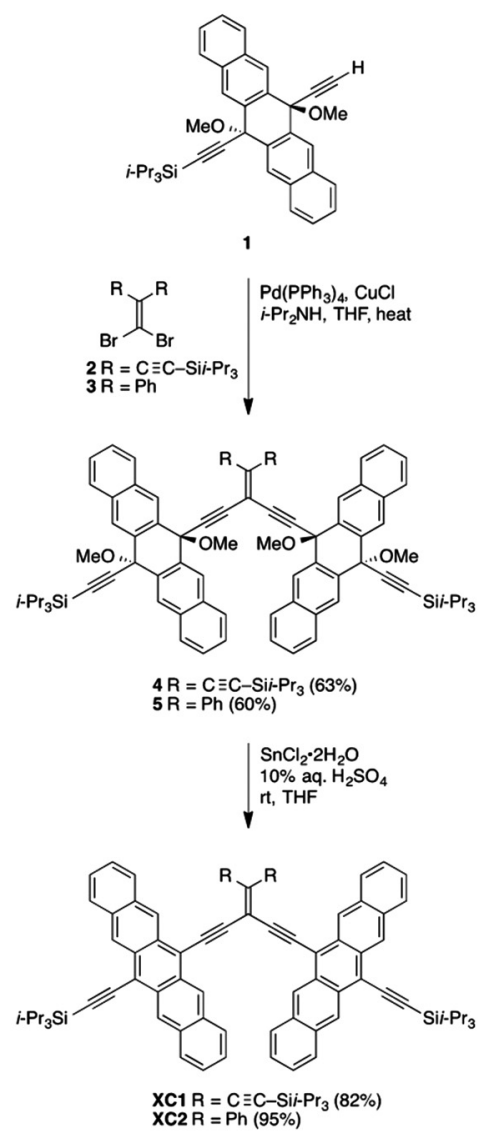

Scheme 1 Synthesis of XC1 and XC2. 
normal laboratory conditions, and they have good solubility in typical organic solvents.

A single crystal of XC2 suitable for X-ray crystallographic analysis was grown from a THF solution layered with $\mathrm{MeOH}$ and allowed to evaporate at $4{ }^{\circ} \mathrm{C}$. The solid-state structure emphasizes the proximity of the two pentacenes. Fig. 2 documents that through space interactions are accessible in both the solid and solution states via carbon-carbon contacts between the two pentacene units as short as $3.34 \AA$ (in the crystal), a result that agrees well with that found theoretically (3.41 ̊, see ESI†).

\section{Singlet and triplet excited state energetics}

We turned to absorption and emission spectroscopies combined with electrochemical analyses to establish the singlet and triplet excited state energetics. In terms of ground state features, we note short wavelength absorptions in the region of 350-500 nm and long wavelength absorptions in the 500-775 nm region (Fig. 3). As a result of the rigid structure of XC1 and XC2, both sets of absorptions show distinct vibrational fine structures. The spectroscopically relevant long wavelength absorptions are at $681 \mathrm{~nm}$ (XC1) and $683 \mathrm{~nm}$ (XC2). In concentration dependent experiments, which obey the Lambert-Beer law for dilute solutions, the following extinction coefficients were determined in benzonitrile: $28800 \mathrm{M}^{-1}$ $\mathrm{cm}^{-1}$ (XC1) and $48900 \mathrm{M}^{-1} \mathrm{~cm}^{-1}$ (XC2). It is notable that the values for XC1 and XC2 are less or exactly twice that of TIPS pentacene 7 (Fig. 4), respectively. A likely rationale infers stronger electronic communications between the two pentacenes in $\mathrm{XC1}$ relative to $\mathrm{XC2}$.

In terms of excited state features, exciting into the long wavelength absorptions leads to fluorescence maxima at 691 and $688 \mathrm{~nm}$ and fluorescence quantum yields of $0.03 \pm 0.01 \%$ and $0.12 \pm 0.04 \%$ for $\mathrm{XC} 1$ and $\mathrm{XC} 2$, respectively, in benzonitrile. Notably, these features are all linked to the singlet excited state with energies of $1.81 \mathrm{eV}$. Finally, no appreciable triplet excited state phosphorescence is observed for XC1 or XC2 in either solutions at room temperature or in frozen media experiments upon excitation in the long wavelength absorption region.

To complement absorption and fluorescence studies, squarewave voltammetric analyses of XC1 and XC2 have been carried out in benzonitrile solutions containing $0.1 \mathrm{M} \mathrm{TBAPF}_{6}$

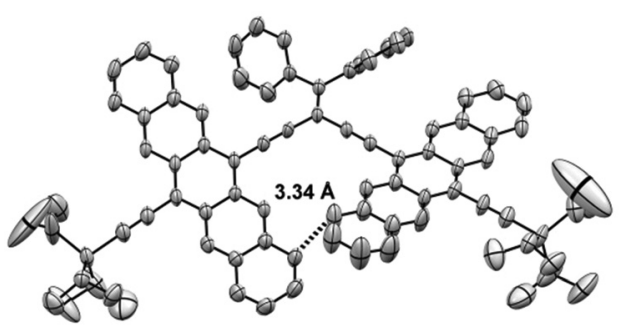

Fig. 2 X-ray crystallographic structure of XC2 illustrating the spatial arrangement and proximity of the two pentacene units in the dimer. THF solvent molecules and hydrogen atoms have been omitted for clarity.
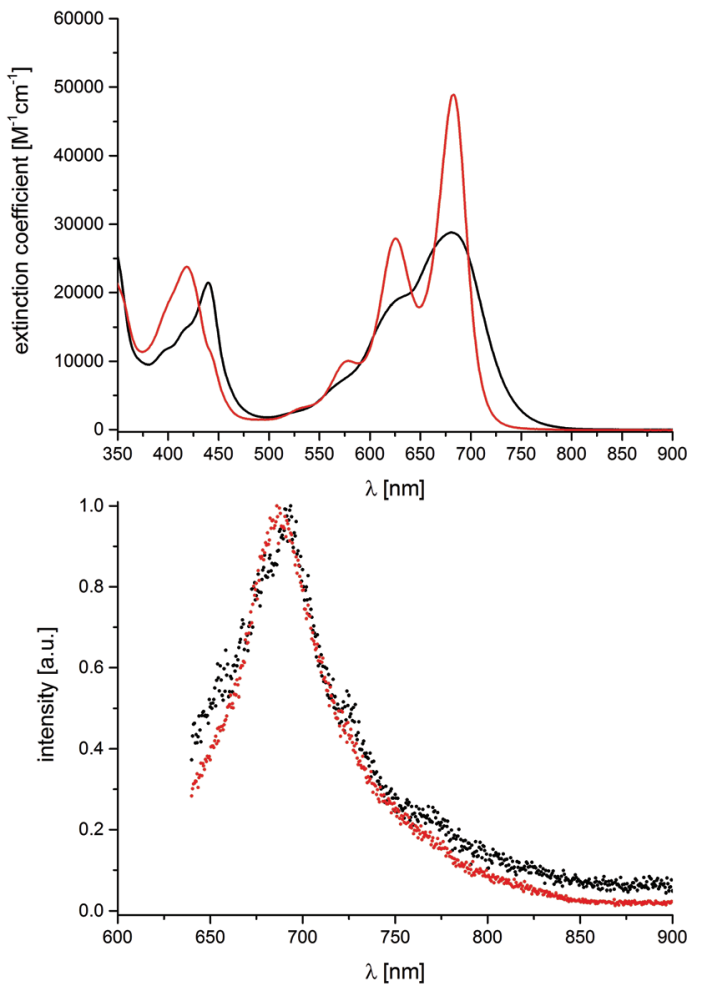

Fig. 3 Top: Room temperature absorption spectra of XC1 (black line) and XC2 (red line) in benzonitrile. Bottom: Normalized fluorescence spectra of XC1 (black dots) and XC2 (red dots) in benzonitrile solutions following photoexcitation at $625 \mathrm{~nm}$ with an optical density of 0.05 . The corresponding fluorescence quantum yields in benzonitrile are $0.03 \pm$ $0.01 \%$ for XC1 and $0.12 \pm 0.04 \%$ for XC2 (measured relative to a zinc phthalocyanine used as a reference compound ${ }^{65}$ ).

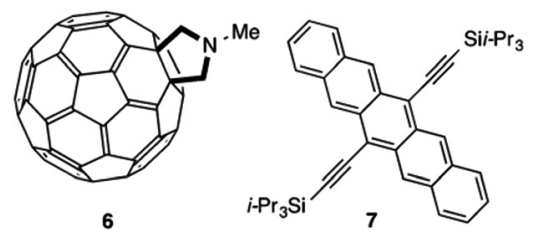

Fig. 4 Molecular structures of $C_{60}$ derivative 6 and TIPS pentacene 7 .

as supporting electrolyte. From the one electron oxidations at +0.44 and $+0.35 \mathrm{~V}$, as well as the one electron reductions at -1.27 and $-1.39 \mathrm{~V}$ for $\mathrm{XC1}$ and $\mathrm{XC2}$ (versus $\mathrm{Fc} / \mathrm{Fc}^{+}$), respectively, highest occupied molecular orbital/lowest unoccupied molecular orbital gaps of 1.71 and $1.74 \mathrm{eV}$ are calculated, which are in sound agreement with the absorption/fluorescence assays.

\section{TIPS pentacene}

First, we examined 7 in 2-methyltetrahydrofuran (Me-THF, $3.0 \times 10^{-4} \mathrm{M}$ ) with excitation at $656 \mathrm{~nm}$ to stimulate population of only vibrational states of the first singlet excited state manifold (Fig. 5). Additionally, the photon flux has been adjusted to $6.6 \times 10^{9}$ photons per pulse to excite, on average, $10 \%$ or less of 7 in its ground state in order to rule out multiple excitations. 


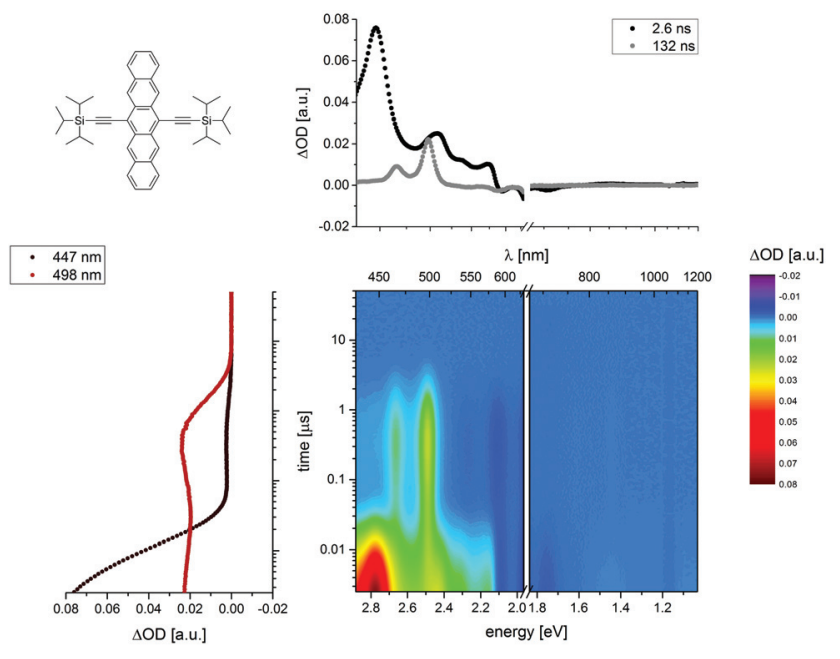

Fig. 5 Upper left: Chemical structure of TIPS pentacene 7. Upper right: Differential absorption spectra (visible and near-infrared) of the spectra shown in the lower right with time delays of 2.6 ns (black) and $132 \mathrm{~ns}$ (gray). Lower left: Time absorption profiles of the spectra shown in the lower right at $447 \mathrm{~nm}$ (black), and $498 \mathrm{~nm}$ (red) illustrating the dynamics of the singlet excited state formation followed by the singlet to triplet transformation in the form of intersystem crossing, and triplet decay. Lower right: Differential absorption changes (visible and near-infrared) obtained upon femtosecond pump probe experiments $(656 \mathrm{~nm})$ of 7 $\left(3.0 \times 10^{-4} \mathrm{M}\right)$ in argon-saturated Me-THF at room temperature with several time delays between 0 and $50 \mu \mathrm{s}$.

For photoexcited 7 , the major deactivation pathway includes a $12.3 \pm 0.1$ ns intersystem crossing, through which the singlet excited state transforms into the corresponding triplet manifold. Once formed, the triplet excited state deactivates with $24.0 \pm 0.5 \mu$ s to reinstate the singlet ground state of 7. As far as the singlet excited state characteristics are concerned, maxima at 447, 508, 538, 572, 845, and $1400 \mathrm{~nm}$ are noted, as well as minima at 595 and $641 \mathrm{~nm}$. An additional feature at $710 \mathrm{~nm}$ is observed, which corresponds to stimulated emission. Triplet excited state characteristics include maxima at 465 and $498 \mathrm{~nm}$ and minima at 546, 587, and $641 \mathrm{~nm}$. Owing to a moderate triplet quantum yield of only $16 \%$ for 7 the resulting characteristics turn out to be extremely weak.

\section{Triplet-triplet sensitization}

To investigate the triplet excited states characteristics via an inefficient intersystem crossing - vide supra - we turned to intermolecular, that is, diffusion or activation controlled, triplet-triplet sensitization experiments using the well-studied $\mathrm{C}_{60}$ derivative 6 as a photosensitizer to examine the triplet excited state characteristics of 7 as well as of XC1 and XC2 (Fig. 4, 6 and S9-S17†). Incentives for the use of 6 are an excitation wavelength independent triplet quantum yield of unity, a triplet excited state energy $1.5 \mathrm{eV}$ higher than that of 7, XC1, and $\mathbf{X C 2}$, a triplet excited state molar extinction coefficient of $16000 \mathrm{M}^{-1} \mathrm{~cm}^{-1}$ at its transient maximum at $695 \mathrm{~nm}$, and a lack of chemical reactions with pentacene derivatives in the
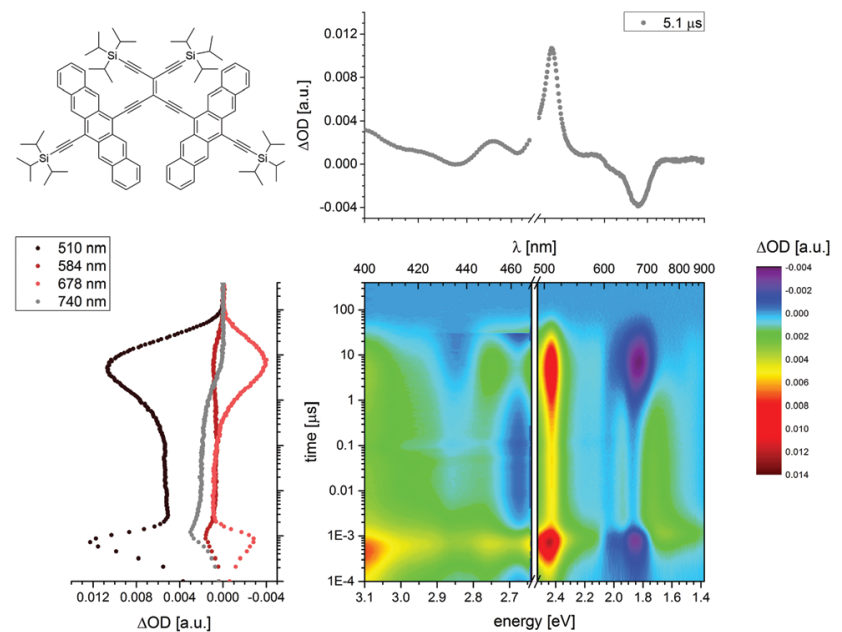

Fig. 6 Upper left: Chemical structure of XC1. Upper right: Differential absorption spectrum (visible and near-infrared) of the spectra shown in the lower right with a time delay of $5.1 \mu \mathrm{s}$ representing the triplet excited state of XC1. Lower left: Time absorption profiles of the spectra shown in the lower right at $510 \mathrm{~nm}$ (black), $584 \mathrm{~nm}$ (red), $678 \mathrm{~nm}$ (orange), and $740 \mathrm{~nm}$ (gray) illustrating the dynamics of the 6 singlet excited state formation followed by the intersystem crossing to the corresponding 6 triplet excited state and transduction of triplet excited state energy to XC1. Lower right: Differential absorption changes (visible and near-infrared) obtained upon femtosecond pump probe experiments $(480 \mathrm{~nm})$ of $6\left(8.0 \times 10^{-5} \mathrm{M}\right)$ and XC1 $\left(1.0 \times 10^{-4} \mathrm{M}\right)$ in argonsaturated toluene at room temperature with several time delays between 0 and $400 \mu \mathrm{s}$.

applied concentration range. ${ }^{66}$ Excitation of 6 at $480 \mathrm{~nm}$ $\left(8.0 \times 10^{-5} \mathrm{M}\right)$ resulted in differential absorption changes on the nanosecond/microsecond time scale featuring a $695 \mathrm{~nm}$ maximum and a $840 \mathrm{~nm}$ shoulder. These originate from a $1.5 \mathrm{~ns}$ intersystem crossing between the corresponding singlet and triplet excited manifolds. In the absence of 7, XC1, XC2, and molecular oxygen, these features decay within $33.0 \pm$ $1.0 \mu \mathrm{s}$ due to triplet-triplet and triplet-ground state annihilations. Addition of 7, XC1, or XC2 in the concentration range from $10^{-5}$ to $10^{-4} \mathrm{M}$ affords nearly diffusion-controlled deactivations of the triplet excited state of 6 with rate constants on the order of $2.5 \times 10^{9} \mathrm{M}^{-1} \mathrm{~s}^{-1} \cdot{ }^{67,68}$ Simultaneously with such a faster decay newly developing transient features are noted including a maximum at $510 \mathrm{~nm}$ and a minimum at $678 \mathrm{~nm}$ for XC1 as well as a maximum at $508 \mathrm{~nm}$ and minima at 622 and $679 \mathrm{~nm}$ for XC2. Here, the excited state minima are precise mirror images of the ground state absorption maxima. Notable is also the close resemblance with the features seen for 7 and several other pentacence dimers, ${ }^{27}$ from which we conclude a transduction of triplet excited state energy to XC1 and XC2. The lifetimes of the triplet excited states of XC1 and XC2 are bimolecular in nature. From the corresponding rate constants, namely $(1.1 \pm 0.1) \times 10^{8}$ and $(0.7 \pm 0.1) \times 10^{8} \mathrm{M}^{-1} \mathrm{~s}^{-1}$ for $\mathbf{X C 1}$ and $\mathbf{X C 2}$, respectively, we deduce activation limited triplet excited state decays including triplet-triplet annihilation as a major component and collision induced triplet-ground state annihilation as a minor component. 
With the triplet excited state molar extinction coefficients of 6 at hand, we calculated the triplet excited state extinction coefficients at the $508 \mathrm{~nm}$ maximum, the $622 \mathrm{~nm}$ minimum, and the $679 \mathrm{~nm}$ minimum at each concentration of XC2, as well as at the $510 \mathrm{~nm}$ maximum and the $678 \mathrm{~nm}$ minimum of XC1. By plotting the extinction coefficients as a function of concentration, we noticed a saturation behavior towards the high concentration regime. At an extrapolation to $5 \times 10^{-4} \mathrm{M}$ the triplet-triplet sensitization is quantitative relative to the initially photoexcited $\mathbf{6}$. Of importance is the fact that the molar extinction coefficients of 21200 and $52800 \mathrm{M}^{-1} \mathrm{~cm}^{-1}$ for XC2 at the $622 \mathrm{~nm}$ minimum and the $679 \mathrm{~nm}$ minimum, respectively, are in sound agreement with the ground state molar extinction coefficients of 30800 and $57900 \mathrm{M}^{-1} \mathrm{~cm}^{-1}$ at 620 and $678 \mathrm{~nm}$, respectively. Likewise the value of $28700 \mathrm{M}^{-1}$ $\mathrm{cm}^{-1}$ for XC1 at its $678 \mathrm{~nm}$ minimum matches that seen at $675 \mathrm{~nm}$ in the ground state absorption. From the aforementioned we infer quantitative bleaching of the ground state for XC1 or XC2 and, in turn, triplet excited states, in which both pentacenes are excited. Considering pseudo first order conditions, namely a laser intensity that is equivalent to approximately $10^{-7} \mathrm{M}$ triplet excited state of 6 and $5.0 \times 10^{-5} \mathrm{M}$ of XC1 or XC2, we postulate that the triplet excited state molar extinction coefficients are only half of the extrapolated values, that is, 45200,10600 and $26400 \mathrm{M}^{-1} \mathrm{~cm}^{-1}$ at the $508 \mathrm{~nm}$ maximum, the $622 \mathrm{~nm}$ minimum, and the $679 \mathrm{~nm}$ minimum, respectively. In fact, with this assumption they are identical to what has been seen for 7, which features molar extinction coefficients about half of that of XC2.

To substantiate our postulate we performed actinometry using pristine $\mathrm{C}_{60} \cdot{ }^{69}$ Here, we used solutions of pristine $\mathrm{C}_{60}$ with four different concentrations and solutions of either 7 , $\mathbf{X C 1}$, or XC2, each of them with exactly the same absorptions at the $387 \mathrm{~nm}$ excitation wavelength, and pumped them with the same laser power. In Fig. $\mathrm{S} 18, \dagger$ the intensities of the $\mathrm{C}_{60}$ singlet excited state markers, on one hand, and the 7, XC1, and XC2 singlet as well as triplet excited state markers, on the other hand, are plotted as a function of absorption. This dependence documents the linear dependence of each excited marker within the range of absorptions. Correlating the respective slopes for pristine $\mathrm{C}_{60}$ with those for 7, XC1, or XC2 enable the determination of the molar extinction coefficients for 7, XC1, and XC2 at, for example, time delays of 1.5 ps. To this end, we determined singlet excited state molar extinction coefficients for XC2 of $14400 \mathrm{M}^{-1} \mathrm{~cm}^{-1}$ at the $622 \mathrm{~nm}$ minimum and of $24100 \mathrm{M}^{-1} \mathrm{~cm}^{-1}$ at the $677 \mathrm{~nm}$ minimum. For XC1, the corresponding singlet excited state molar extinction coefficient is $20200 \mathrm{M}^{-1} \mathrm{~cm}^{-1}$ at $679 \mathrm{~nm}$. All of these values relate to half of the ground state extinction coefficients/ ground state bleaching and suggest singlet excited states, in which only a single pentacene is photoexcited. The latter assumption is in sound agreement with the low laser flux of $6.6 \times 10^{9}$ photons per pulse. Additional support was provided by the fact that the $647 \mathrm{~nm}$ value of $15000 \mathrm{M}^{-1} \mathrm{~cm}^{-1}$ for 7 correlates quantitatively with the ground state extinction coefficient/ground state bleaching. In case that 7, XC1, and XC2 show quantitative intersystem crossing, that is, affording triplet quantum yields of $100 \%$, the triplet excited state molar extinction coefficients at 647, 679, and $677 \mathrm{~nm}$, respectively, should match those of the singlet excited states.

\section{Singlet fission in XC1 and XC2}

For XC2 in benzonitrile, the singlet excited state features are discernable as a $455 \mathrm{~nm}$ maximum and a broad band in the NIR as well as minima at 627 and $687 \mathrm{~nm}$ directly upon photoexcitation at $656 \mathrm{~nm}$ (Fig. 7). In contrast to that observed for 7, where a slow intersystem crossing dominates the initial $20 \mathrm{~ns}$, the singlet excited state features of XC2 are replaced within 15 ps by characteristics that match those seen in the triplettriplet sensitization experiments (Fig. S17†). In particular, these are maxima at 486, 517, 853, and $976 \mathrm{~nm}$ and minima at 628 and 685 nm. From multiwavelength analyses, which afforded strictly unimolecular, intramolecular dynamics in the range from $1 \times 10^{-5}$ to $1 \times 10^{-4} \mathrm{M}$ of $\mathbf{X C 2}$, we conclude that these features correspond to the rapidly decaying singlet excited state $\left((0.7 \pm 0.1) \times 10^{12} \mathrm{~s}^{-1}\right)$ and the simultaneously grow of the triplet excited state markers. Please note that there is a slight blue shift, from 687 to $685 \mathrm{~nm}$, as a consequence of this transformation. A closer look at delay times up to $2000 \mathrm{ps}$, however, reveals the metastability of the newly formed triplet excited state. Considering, on one hand, that the experimental conditions preclude multiple excitations of XC2 and, on the other hand, that triplet-triplet annihilation contributes most strongly to the triplet excited state decay we tested the

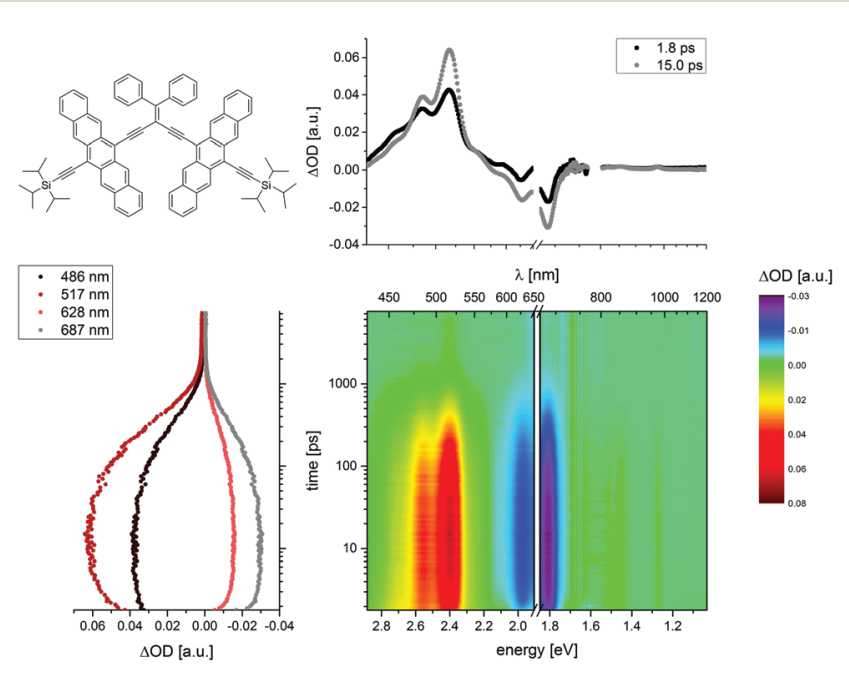

Fig. 7 Upper left: Chemical structure of XC2. Upper right: Differential absorption spectra (visible and near-infrared) of the spectra shown in the lower right with time delays of $1.8 \mathrm{ps}$ (black) and $15.0 \mathrm{ps}$ (gray). Lower left: Time absorption profiles of the spectra shown in the lower right at $486 \mathrm{~nm}$ (black), $517 \mathrm{~nm}$ (red), $628 \mathrm{~nm}$ (orange), and $687 \mathrm{~nm}$ (gray) illustrating the dynamics of the singlet excited state formation followed by the singlet to triplet transformation in the form of singlet fission and triplet-triplet annihilation. Lower right: Differential absorption changes (visible and near-infrared) obtained upon femtosecond pump probe experiments $(656 \mathrm{~nm})$ of XC2 $\left(5.0 \times 10^{-5} \mathrm{M}\right)$ in argon-saturated benzonitrile at room temperature with several time delays between 0 and 7500 ps. 
impact of different concentrations in the range from $1 \times 10^{-5}$ to $1 \times 10^{-4} \mathrm{M}$. Regardless of the concentration, we note only unimolecular and invariant dynamics with a rate constant of $(2.54 \pm 0.08) \times 10^{9} \mathrm{~s}^{-1}$. As such, we hypothesize unimolecular triplet-triplet annihilations, which requires that two triplets are localized within XC2 and this is only feasible by SF - vide infra. Spectroscopic corroboration for two photoexcited pentacenes came from a $2-3 \mathrm{~nm}$ blue-shift not only relative to the singlet excited state bleaching but also relative to the triplet excited state bleaching seen in direct excitation and triplettriplet sensitization experiments, respectively. The product of the triplet-triplet annihilations is the singlet ground state of XC2 via radiationless relaxation, ${ }^{70}$ and independent on concentration or laser power. But, a spectroscopically invisible excited state cannot be ruled out. As a complement to multiwavelength analyses, we performed global analyses with the Glotaran software package (Fig. 9). ${ }^{71}$ The results, as they were obtained from the global fitting, are a qualitative and quantitative match to those gathered in the multiwavelength approach for XC2 and XC1. For example, the singlet excited state in XC2 transforms sequentially into the triplet excited state and ground state within $0.7 \pm 0.1$ and $445 \pm 20$ ps, respectively.

Very similar observations are made for XC1 in benzonitrile (Fig. 8 and 9), in terms of differential absorption changes and kinetics versus XC2. In particular, the singlet excited state for XC1 converts with similar decay and growth kinetics of $(0.65 \pm$
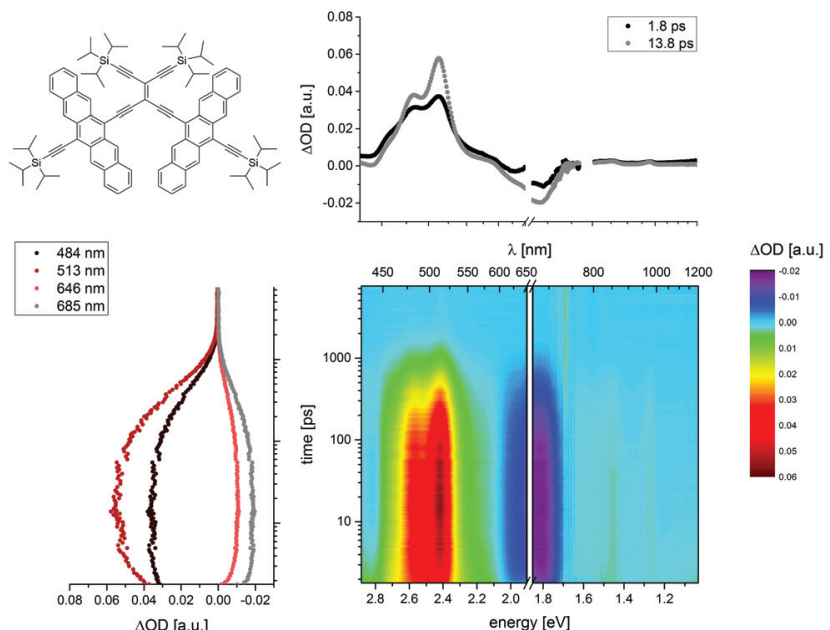

Fig. 8 Upper left: Chemical structure of XC1. Upper right: Differential absorption spectra (visible and near-infrared) of the spectra shown in the lower right with time delays of 1.8 ps (black) and 13.8 ps (gray). Lower left: Time absorption profiles of the spectra shown in the lower right at $484 \mathrm{~nm}$ (black), $513 \mathrm{~nm}$ (red), $646 \mathrm{~nm}$ (orange), and $685 \mathrm{~nm}$ (gray) illustrating the dynamics of the singlet excited state formation followed by the singlet to triplet transformation in the form of singlet fission and triplet-triplet annihilation. Lower right: Differential absorption changes (visible and near-infrared) obtained upon femtosecond pump probe experiments $(656 \mathrm{~nm})$ of XC1 $\left(5.0 \times 10^{-5} \mathrm{M}\right)$ in argon-saturated benzonitrile at room temperature with several time delays between 0 and 7500 ps.
$0.1) \times 10^{12} \mathrm{~s}^{-1}$ via SF into a metastable triplet excited state. In THF and toluene the corresponding kinetics tend to be as slow as $(0.63 \pm 0.1) \times 10^{12}$ and $(0.50 \pm 0.1) \times 10^{12} \mathrm{~s}^{-1}$. Analyses of the long wavelength minima reveals a blue-shift of $2-3 \mathrm{~nm}$ when comparing the singlet and triplet excited state spectra. Similar to $\mathbf{X C 2}$, the metastability observed for $\mathbf{X C 1}$ is seen in the form of unimolecular, intramolecular triplet-triplet annihilations $\left((2.08 \pm 0.07) \times 10^{9} \mathrm{~s}^{-1}\right)$. The latter results in fast and quantitative ground state recovery on a time scale of less than 3000 ps. Resembling XC2, the two steps, namely SF and the decay of the correspondingly formed triplet excited state, are clearly deconvoluted. If at all, the different cross conjugation in XC2 results in slightly faster triplet excited state formation and slightly faster triplet excited state decay relative to XC1, but the differences are small. The absorption spectra - vide supra point possibly to stronger electronic coupling in XC1, which is likely derived from through-bond interactions. As such, we hypothesize that solution SF seems more favorable in the latter scenario.

To corroborate SF as the mechanism for singlet to triplet conversion we have used three different approaches, that is, the sensitization method, the bleaching method, and the actinometry method, to determine the triplet quantum yields. In the first approach, 6 is used as an external reference to derive the transient extinction coefficients. Firstly, we have revisited the triplet-triplet sensitization experiments, whose differential absorption spectra in the high concentration regime, where the triplet energy transfer efficiency is close to unity, enable derivation of triplet molar extinction coefficients for XC2 of 45200,10600 and $26400 \mathrm{M}^{-1} \mathrm{~cm}^{-1}$ at the $508 \mathrm{~nm}$ maximum, the $622 \mathrm{~nm}$ minimum, and the $679 \mathrm{~nm}$ minimum, respectively. Next, we have determined the singlet molar extinction coefficients with the help of conventional femtosecond transient absorption measurements. XC2, for example, features only very weak ground state absorption at $456 \mathrm{~nm}$ in benzonitrile, and, as such, we take the amplitude of the differential absorptions at $455 \mathrm{~nm}$ after $1 \mathrm{ps}$ and relate it entirely to the singlet excited state concentration formed upon excitation. Finally, the differential absorption changes of 0.0242 a.u. at $455 \mathrm{~nm}$ and 0.0679 a.u. at $518 \mathrm{~nm}$ are converted into concentrations of the singlet excited state of $4.64 \times 10^{-6} \mathrm{M}$ and of the triplet excited state of $7.51 \times 10^{-6} \mathrm{M}$. This ultimately converts to a triplet quantum yield of $162 \pm 10 \%$ for XC2.

Independent confirmation of the triplet quantum yield comes from the second approach (bleaching method), in which no external reference is used to calculate the transient extinction coefficients of the singlet and triplet excited state. In this case, the amplitudes of the ground state bleaching in benzonitrile at $687 \mathrm{~nm}(-0.0245 \mathrm{a} . \mathrm{u})$ and $685 \mathrm{~nm}(-0.0314$ a.u. $)$ are used to calculate the transient concentrations as $4.64 \times 10^{-6}$ and $5.96 \times 10^{-6} \mathrm{M}$ for the singlet and the triplet excited state, respectively. This affords a triplet quantum yield of $128 \pm 5 \%$ for XC2. The high triplet quantum yield along with its ultrafast formation provide strong evidence that the triplet excited state for XC2 forms by intramolecular SF between the two pentacenes. Moreover, higher quantum yields in more polarizable solvents - 

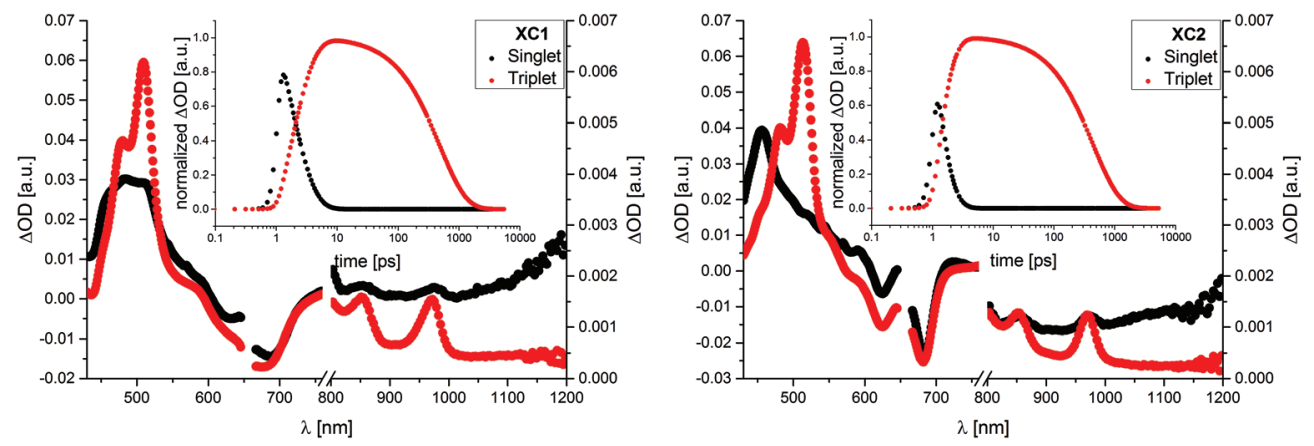

Fig. 9 Deconvoluted transient absorption spectra of the singlet (black) and the triplet excited state (red) of XC1 and XC2 as obtained by global analysis (visible and near-infrared). Inset: Evolution of the population of the singlet (black) and the triplet excited state (red).

mean values of $128 \pm 10 \%$ in toluene versus $162 \pm 10 \%$ in benzonitrile - corroborate the charge transfer nature of the mediating step. Analogously, the triplet quantum yields for XC1 are derived as $127 \pm 10 \%$ with an external reference and $124 \pm 5 \%$ without an external reference in benzonitrile. In toluene, the corresponding yields for XC1 are lower with values of $119 \pm$ $10 \%$ and $114 \pm 5 \%$, respectively (Table 1$).{ }^{72}$ In a third approach we used the results from the actinometry experiments to calculate the triplet quantum yields. Correlating the slopes for the respective singlet and triplet excited state features enable the determination of triplet quantum yields of $111 \pm 5 \%$ for XC1 and $107 \pm 5 \%$ for XC2 in toluene, in good agreement with the results obtained by means of the first and second approach.

\section{Theory}

To further solidify the experimental results, we have investigated the electronic states of relevance for SF using multireference perturbation theory methods (see ESI $\dagger$ for details). The calculated vertical excitation energies, dipole moments, oscillator strengths, and character of the relevant adiabatic states are summarized in Table 2. For XC1 and XC2, the ME state is the lowest-lying singlet excited state (see Table 2 and ESI $\dagger$ ). Furthermore, the vertical excitation energies of the lowest-lying absorbing $\mathrm{S}_{2}$ state of XC1 $(1.88 \mathrm{eV})$ and XC2 $(1.90 \mathrm{eV})$ agree well with the experimental data. Both XC1 and XC2 exhibit CT states $\left(\mathrm{S}_{4}\right.$ and $\left.\mathrm{S}_{5}\right)$ with moderate dipole moments that are relatively high in energy $(\sim 0.5-0.6 \mathrm{eV})$ compared to the $S_{2}$ state.

Table 1 Triplet excited state quantum yields $\left(\Phi_{\text {Triplet }}\right)$ for XC1 and XC2 calculated via the sensitization method, the bleaching method, and the actinometry

\begin{tabular}{lllll}
\hline & & $\begin{array}{l}\text { Sensitization } \\
\text { method }\end{array}$ & $\begin{array}{l}\text { Bleaching } \\
\text { method }\end{array}$ & Actinometry \\
\cline { 3 - 5 } & & Triplet quantum yield [\%] & \\
\hline $\mathbf{X C 1}$ & Toluene & 119 & 114 & 111 \\
& THF & 131 & 121 & - \\
$\mathbf{X C 2}$ & Benzonitrile & 127 & 124 & - \\
& Toluene & 128 & 109 & 107 \\
& THF & 144 & 113 & - \\
& Benzonitrile & 162 & 128 & -
\end{tabular}

To shed light on the SF mechanism, we have determined the diabatic electronic states and their couplings employing Truhlar's four-fold-way diabatization procedure (see ESI $\dagger$ ). ${ }^{73,74}$ We report in the following the results obtained for XC1 and XC2. Following previous works, ${ }^{2,18,60}$ the diabatic states are classified as the singlet ground state $\left|S_{0} S_{0}\right\rangle$, the correlated triplet pair (or $\mathrm{ME}$ ) state $\left|\mathrm{T}_{1} \mathrm{~T}_{1}\right\rangle$, the locally excited (in one pentacene chromophore) singlet states $\left|S_{1} S_{0}\right\rangle$ and $\left|S_{0} S_{1}\right\rangle$ and the charge transfer states $|\mathrm{CA}\rangle$ and $|\mathrm{AC}\rangle$. In the latter, one of the two pentacenes is formally a radical cation (C), while the other is a radical anion (A). For XC1 and XC2, the diabatic coupling matrices are presented in Table 3. As Table 3 documents, XC1 and XC2 each exhibit a moderate electronic coupling between the locally excited states $\left(\left|S_{1} S_{0}\right\rangle\right.$ and $\left.\left|S_{0} S_{1}\right\rangle\right)$ and $\left|T_{1} T_{1}\right\rangle$. Specifically, $\left|S_{1} S_{0}\right\rangle$ and $\left|S_{0} S_{1}\right\rangle$ couple to $\left|T_{1} T_{1}\right\rangle$ with a strength of 9 and $-12 \mathrm{meV}$, respectively, in XC1. In XC2, the corresponding values are -1 and $2 \mathrm{meV}$. In contrast, the electronic coupling between $\left|\mathrm{S}_{1} \mathrm{~S}_{0}\right\rangle$ or $\left|\mathrm{S}_{0} \mathrm{~S}_{1}\right\rangle$, and the charge transfer states $|\mathrm{CA}\rangle$ and $|\mathrm{AC}\rangle$ range from 30 to $140 \mathrm{meV}$. As such, this is nearly an order of magnitude larger than that between the locally excited and the correlated triplet pair state $\left|\mathrm{T}_{1} \mathrm{~T}_{1}\right\rangle$ (Table 3). $\left|\mathrm{T}_{1} \mathrm{~T}_{1}\right\rangle$ also couples to the $|\mathrm{CA}\rangle$ and $|\mathrm{AC}\rangle$ states with 10-60 meV in XC1 and 23-27 meV in XC2.

To dissect contributions from either the direct or the mediated SF mechanism and the role of CT states, we estimated the magnitude of the effective coupling, $V_{\text {eff }}$, of the lowest lying absorbing singlet state $\left|\mathrm{S}_{1} \mathrm{~S}_{0}\right\rangle$ to $\left|\mathrm{T}_{1} \mathrm{~T}_{1}\right\rangle$ (similar for $\left|\mathrm{S}_{0} \mathrm{~S}_{1}\right\rangle$ to $\left.\left|\mathrm{T}_{1} \mathrm{~T}_{1}\right\rangle\right)$ as: ${ }^{55,75}$

$$
V_{\mathrm{eff}} \approx V_{\mathrm{S}_{1} \mathrm{~S}_{0}, \mathrm{~T}_{1} \mathrm{~T}_{1}}-2 \frac{V_{\mathrm{S}_{1} \mathrm{~S}_{0}, \mathrm{CA}} V_{\mathrm{CA}, \mathrm{T}_{1} \mathrm{~T}_{1}}+V_{\mathrm{S}_{1} \mathrm{~S}_{0}, \mathrm{AC}} V_{\mathrm{AC}, \mathrm{T}_{1} \mathrm{~T}_{1}}}{\left[E(\mathrm{CT})-E\left(\mathrm{~T}_{1} \mathrm{~T}_{1}\right)\right]+\left[E(\mathrm{CT})-E\left(\mathrm{~S}_{1} \mathrm{~S}_{0}\right)\right]}
$$

where $E(i)$ are the energies of the corresponding diabatic states, $E(\mathrm{CT})$ is the mean of $E(\mathrm{CA})$ and $E(\mathrm{AC})$, and $V_{i, j}$ are the couplings between the states $i$ and $j$. The first term in eqn (1) denotes the direct coupling and the second term the indirect coupling mediated by the CT states. The results in Table 4 indicate that in both XC1 and XC2 the direct coupling of $\left|\mathrm{S}_{1} \mathrm{~S}_{0}\right\rangle$ to $\left|\mathrm{T}_{1} \mathrm{~T}_{1}\right\rangle$ is significantly smaller than the indirect coupling via CT states. The differences between both types of coupling are smaller in the case of $\mathbf{X C 2}$ relative to $\mathbf{X C 1}$. For the second bright state $\left|S_{0} S_{1}\right\rangle$, which is quasi-degenerate to $\left|S_{1} S_{0}\right\rangle$, 
Table 2 Vertical excitation energy $(\Delta E$ in $\mathrm{eV}),{ }^{a}$ modulus of the dipole moment $(\mu, \mathrm{D}),{ }^{b}$ oscillator strength $(f),{ }^{b}$ and character for the lowest lying singlet excited states $(\text { char. })^{c}$ of XC1 and XC2

\begin{tabular}{|c|c|c|c|c|c|c|c|c|}
\hline State & \multicolumn{4}{|l|}{ XC1 } & \multicolumn{4}{|l|}{ XC2 } \\
\hline $\mathrm{S}_{2}$ & 1.88 & 4.43 & 0.880 & LE & 1.90 & 1.74 & 0.770 & LE \\
\hline $\mathrm{S}_{3}$ & 1.94 & 3.39 & 0.610 & $\mathrm{LE}$ & 1.95 & 1.74 & 0.640 & LE \\
\hline $\mathrm{S}_{4}$ & 2.31 & 8.23 & 0.004 & $\mathrm{CT}$ & 2.36 & 8.31 & 0.005 & $\mathrm{CT}$ \\
\hline $\mathrm{S}_{5}$ & 2.40 & 8.79 & 0.002 & CT & 2.40 & 8.33 & 0.001 & CT \\
\hline
\end{tabular}

${ }^{a}$ Calculated at the XMCQDPT/DZV level of theory. ${ }^{b}$ Calculated at the CASSCF level of theory. ${ }^{c}$ Character of excited state: ME $=$ multiexcitonic state, $\mathrm{LE}=$ excited states that correlate with the plus and minus combinations of locally excited states of both pentacene monomers, CT $=$ charge transfer states.

Table 3 Energies and coupling matrix elements (meV) of the low-lying diabatic electronic states of XC1 (top) and XC2 (bottom) calculated at the XMCQDPT/DZV level of theory

\begin{tabular}{lllllll}
\hline $\mathbf{X C 1}$ & $\left|\mathrm{S}_{0} \mathrm{~S}_{0}\right\rangle$ & $\left|\mathrm{T}_{1} \mathrm{~T}_{1}\right\rangle$ & $\left|\mathrm{S}_{1} \mathrm{~S}_{0}\right\rangle$ & $\left|\mathrm{S}_{0} \mathrm{~S}_{1}\right\rangle$ & $|\mathrm{CA}\rangle$ & $|\mathrm{AC}\rangle$ \\
\hline$\left|\mathrm{S}_{0} \mathrm{~S}_{0}\right\rangle$ & 0 & 10 & 261 & 105 & 81 & 18 \\
$\left|\mathrm{~T}_{1} \mathrm{~T}_{1}\right\rangle$ & 10 & 1779 & 9 & -12 & 61 & 11 \\
$\left|\mathrm{~S}_{1} \mathrm{~S}_{0}\right\rangle$ & 261 & 9 & 1863 & -29 & 141 & 71 \\
$\left|\mathrm{~S}_{0} \mathrm{~S}_{1}\right\rangle$ & 105 & -12 & -29 & 1885 & -31 & 34 \\
$|\mathrm{CA}\rangle$ & 81 & 61 & 141 & -31 & 2217 & 21 \\
$|\mathrm{AC}\rangle$ & 18 & 11 & 71 & 34 & 21 & 2323 \\
\hline $\mathbf{X C 2}$ & $\left|\mathrm{S}_{0} \mathrm{~S}_{0}\right\rangle$ & $\left|\mathrm{T}_{1} \mathrm{~T}_{1}\right\rangle$ & $\left|\mathrm{S}_{1} \mathrm{~S}_{0}\right\rangle$ & $\left|\mathrm{S}_{0} \mathrm{~S}_{1}\right\rangle$ & $|\mathrm{CA}\rangle$ & $|\mathrm{AC}\rangle$ \\
\hline$\left|\mathrm{S}_{0} \mathrm{~S}_{0}\right\rangle$ & 0 & -13 & -119 & 139 & -140 & -118 \\
$\left|\mathrm{~T}_{1} \mathrm{~T}_{1}\right\rangle$ & -13 & 1806 & -1 & 2 & -27 & 23 \\
$\left|\mathrm{~S}_{1} \mathrm{~S}_{0}\right\rangle$ & -119 & -1 & 2009 & -94 & -136 & -72 \\
$\left|\mathrm{~S}_{0} \mathrm{~S}_{1}\right\rangle$ & 139 & 2 & -94 & 2025 & 138 & 87 \\
$|\mathrm{CA}\rangle$ & -140 & -27 & -136 & 138 & 2172 & -130 \\
$|\mathrm{AC}\rangle$ & -118 & 23 & -72 & 87 & -130 & 2282
\end{tabular}

Table 4 Direct, indirect, and total coupling (meV) of the lowest lying absorbing singlet states $\left|S_{1} S_{0}\right\rangle$ and $\left|S_{0} S_{1}\right\rangle$ to $\left|T_{1} T_{1}\right\rangle$ for XC1 and XC2, calculated at the XMQCDPT/DZV level of theory using Truhlar's four-foldway diabatization method

\begin{tabular}{llrrr}
\hline System & State & Direct & Indirect & Total \\
\hline XC1 & $\left|\mathrm{S}_{1} \mathrm{~S}_{0}\right\rangle$ & 9.39 & -23.69 & -14.30 \\
& $\left|\mathrm{~S}_{0} \mathrm{~S}_{1}\right\rangle$ & -12.09 & 3.94 & -8.15 \\
XC2 & $\left|\mathrm{S}_{1} \mathrm{~S}_{0}\right\rangle$ & -0.83 & -7.62 & -8.45 \\
& $\left|\mathrm{~S}_{0} \mathrm{~S}_{1}\right\rangle$ & 1.78 & 6.72 & 8.50
\end{tabular}

XC1 exhibits an effective coupling smaller than that found for $\left|\mathrm{S}_{1} \mathrm{~S}_{0}\right\rangle$ and where the major contribution stems from the direct coupling of $\left|S_{0} S_{1}\right\rangle$ to $\left|T_{1} T_{1}\right\rangle$. On the other hand, XC2 shows an effective coupling between $\left|S_{1} S_{0}\right\rangle$ and $\left|T_{1} T_{1}\right\rangle$ where the major contribution corresponds to the indirect coupling via CT states. Considering that the CT states are higher in energy than the absorbing states we postulate that SF is likely to proceed predominantly via a superexchange-like mechanism in both molecular systems, although in the case of XC1 we expect a more significant contribution of the direct mechanism to the overall process than in XC2 (see Table 4). This is further corroborated by the experimentally found dependence of the triplet quantum yield on the polarizability of the solvents employed. The results in Table 4 also indicate that the coupling is somewhat larger in XC1 compared to XC2, which may explain the stronger broadening of the absorption spectrum of XC1 (see Fig. 3).

\section{Conclusions}

We have designed cross-conjugated pentacene dimers, XC1 and $\mathbf{X C 2}$, to fine-tune the electronic coupling element between the two pentacenes and to gain control over intramolecular SF in room temperature solutions. To this end, XC1 features slightly stronger electronic coupling, governed by throughbond interactions, than XC2. Kinetic and spectroscopic analyses were based on applications of multiwavelength analysis, on one hand, and global analysis using the program Glotaran, on the other hand. Both led to identical results and corroborate that the cross-conjugated tethers maintain the electronic couplings lending, however, to slightly higher triplet quantum yields for XC2 than for XC1. As a matter of fact, the highest triplet quantum yield observed is $162 \pm 10 \%$.

Our synthetic, spectroscopic, and computational efforts highlight the importance of establishing the right balance between triplet excited state formation/singlet excited state deactivation and triplet excited state decay towards the optimization of SF. An outstanding question remains the loss mechanism(s) from the singlet state in our dimers, and ongoing work in our laboratories focuses on realizing SF quantum yields close to unity through further controlling intramolecular interactions in synthetically designed dimers.

\section{Experimental section}

The synthesis of XC1 and XC2, materials, general methods, and computational details are described in the ESI $\dagger$.

For the photophysical characterization (see ESI $\dagger$ ), the samples were placed in fluorometric cuvettes with different pathways and, when necessary, purged with argon. Steady-state UV-vis absorption spectra were acquired at room temperature (RT) using a Perkin Elmer Lambda 2 spectrometer. Steady- 
state fluorescence spectra were carried out at a FluoroMax3 spectrometer from Horiba in the visible detection range (RT) and at a FluoroLog3 spectrometer from Horiba in the near infrared. Femtosecond (fs) transient absorption experiments were carried out with an amplified Ti:Sapphire CPA-2110 fs laser system (ClarkMXR: output 775 nm, $1 \mathrm{kHz}, 150$ fs pulse width) using transient absorption pump/probe detection systems (Helios and Eos, Ultrafast Systems).

The theoretical characterization was based on the following methods. The ground state equilibrium geometries of XC1 and XC2 were optimized using density functional theory (DFT), ${ }^{76}$ employing the B3LYP correlation-exchange functional and the def2-TZVP basis set. ${ }^{77,78}$ Dispersion interactions were taken into account using Grimme's empirical dispersion correction. ${ }^{78}$ Vertical excitation energies were calculated using the extended multi-configurational quasi-degenerate perturbation theory $(\mathrm{XMCQDPT})^{79}$ method and a double- $\zeta$ basis set, ${ }^{80}$ employing state average (SA) complete active space self-consistent field (CASSCF $)^{81,82}$ wave-functions as reference and an intruder state avoidance shift of $0.02 \mathrm{au} .^{83}$ The active space employed in the SA-CASSCF calculations comprised four electrons in four orbitals (HOMO and LUMO per pentacene unit) and six roots with equal weights were used. Dipole moments and oscillator strengths were calculated at the SA-CASSCF level. Diabatic states were built using Truhlar's four-fold-way of diabatization method implemented in GAMESS. ${ }^{73,74,84}$ All calculations were carried out using the electronic structure packages TURBOMOLE ${ }^{85}$ and GAMESS. ${ }^{86}$

\section{Acknowledgements}

We thank Dr R. McDonald for solving the crystal structure of XC2. Generous allocation of computing time at the computing centers Erlangen (Regionales Rechenzentrum Erlangen), Munich (Leibniz-Rechenzentrum der Bayerischen Akademie der Wissenschaften in München), and Jülich (Jülich Supercomputing Centre) is gratefully acknowledged. Funding is gratefully acknowledged from the Emerging Fields initiative "Singlet Fission" supported by Friedrich-Alexander-Universität Erlangen-Nürnberg, the German Research Foundation (DFG), as well as the Cluster of Excellence Engineering of Advanced Materials and "Solar Technologies Go Hybrid" - an initiative of the Bavarian State Ministry for Science, Research, and Art.

\section{Notes and references}

1 J. C. Johnson, A. J. Nozik and J. Michl, Acc. Chem. Res., 2013, 46, 1290-1299.

2 M. B. Smith and J. Michl, Chem. Rev., 2010, 110, 6891-6936.

3 W. Shockley and H. J. Queisser, J. Appl. Phys., 1961, 32, 510-519.

4 L. Yang, M. Tabachnyk, S. L. Bayliss, M. L. Böhm, K. Broch, N. C. Greenham, R. H. Friend and B. Ehrler, Nano Lett., 2015, 15, 354-358.
5 W. L. Chan, M. Ligges and X. Y. Zhu, Nat. Chem., 2012, 4, 840-845.

6 D. N. Congreve, J. Lee, N. J. Thompson, E. Hontz, S. R. Yost, P. D. Reusswig, M. E. Bahlke, S. Reineke, T. Van Voorhis and M. A. Baldo, Science, 2013, 340, 334-337.

7 T. C. Berkelbach, M. S. Hybertsen and D. R. Reichman, J. Chem. Phys., 2013, 138, 114102.

8 G. B. Piland, J. J. Burdett, R. J. Dillon and C. J. Bardeen, J. Phys. Chem. Lett., 2014, 5, 2312-2319.

9 W. L. Chan, T. C. Berkelbach, M. R. Provorse, N. R. Monahan, J. R. Tritsch, M. S. Hybertsen, D. R. Reichman, J. Gao and X. Y. Zhu, Acc. Chem. Res., 2013, 46, 1321-1329.

10 J. J. Burdett and C. J. Bardeen, Acc. Chem. Res., 2013, 46, 1312-1320.

11 C. E. Swenberg and W. T. Stacy, Chem. Phys. Lett., 1968, 2, 327-328.

12 T. C. Berkelbach, M. S. Hybertsen and D. R. Reichman, J. Chem. Phys., 2014, 141, 074705.

13 W. G. Albrecht, M. E. Michel-Beyerle and V. Yakhot, J. Lumin., 1979, 20, 147-149.

14 A. Rao, M. W. B. Wilson, S. Albert-Seifried, R. Di Pietro and R. H. Friend, Phys. Rev. B: Condens. Matter, 2011, 84, 195411.

15 W. L. Chan, M. Ligges, A. Jailaubekov, L. Kaake, L. MiajaAvila and X. Y. Zhu, Science, 2011, 334, 1541-1545.

16 C. J. Bardeen, Annu. Rev. Phys. Chem., 2014, 65, 127148.

17 N. Monahan and X.-Y. Zhu, Annu. Rev. Phys. Chem., 2015, 66, 601-618.

18 M. B. Smith and J. Michl, Annu. Rev. Phys. Chem., 2013, 64, 361-386.

19 R. E. Merrifield, P. Avakian and R. P. Groff, Chem. Phys. Lett., 1969, 3, 155-157.

20 J. J. Burdett, A. M. Müller, D. Gosztola and C. J. Bardeen, J. Chem. Phys., 2010, 133, 144506.

21 C. Jundt, G. Klein, B. Sipp, J. Le Moigne, M. Joucla and A. A. Villaeys, Chem. Phys. Lett., 1995, 241, 84-88.

22 M. Rei Vilar, M. Heyman and M. Schott, Chem. Phys. Lett., 1983, 94, 522-526.

23 E. Busby, T. C. Berkelbach, B. Kumar, A. Chernikov, Y. Zhong, H. Hlaing, X. Y. Zhu, T. F. Heinz, M. S. Hybertsen, M. Y. Sfeir, D. R. Reichman, C. Nuckolls and O. Yaffe, J. Am. Chem. Soc., 2014, 136, 10654-10660.

24 J. Lee, M. J. Bruzek, N. J. Thompson, M. Y. Sfeir, J. E. Anthony and M. A. Baldo, Adv. Mater., 2013, 25, 14451448.

25 A. M. Müller, Y. S. Avlasevich, W. W. Schoeller, K. Müllen and C. J. Bardeen, J. Am. Chem. Soc., 2007, 129, 1424014250.

26 B. J. Walker, A. J. Musser, D. Beljonne and R. H. Friend, Nat. Chem., 2013, 5, 1019-1024.

27 J. Zirzlmeier, D. Lehnherr, P. B. Coto, E. T. Chernick, R. Casillas, B. S. Basel, M. Thoss, R. R. Tykwinski and D. M. Guldi, Proc. Natl. Acad. Sci. U. S. A., 2015, 112, 53255330. 
28 S. N. Sanders, E. Kumarasamy, A. B. Pun, M. T. Trinh, B. Choi, J. Xia, E. J. Taffet, J. Z. Low, J. R. Miller, X. Roy, X. Y. Zhu, M. L. Steigerwald, M. Y. Sfeir and L. M. Campos, J. Am. Chem. Soc., 2015, 137, 8965-8972.

29 J. E. Anthony, Chem. Rev., 2006, 106, 5028-5048.

30 M. W. Wilson, A. Rao, J. Clark, R. S. Kumar, D. Brida, G. Cerullo and R. H. Friend, J. Am. Chem. Soc., 2011, 133, 11830-11833.

31 K. von Burg and I. Zschokke-Gränacher, J. Chem. Phys., 1979, 70, 3807-3811.

32 P. M. Zimmerman, Z. Zhang and C. B. Musgrave, Nat. Chem., 2010, 2, 648-652.

33 S. R. Yost, J. Lee, M. W. B. Wilson, T. Wu, D. P. McMahon, R. R. Parkhurst, N. J. Thompson, D. N. Congreve, A. Rao, K. Johnson, M. Y. Sfeir, M. G. Bawendi, T. M. Swager, R. H. Friend, M. A. Baldo and T. Van Voorhis, Nat. Chem., 2014, 6, 492-497.

34 L. Wang, Y. Olivier, O. V. Prezhdo and D. Beljonne, J. Phys. Chem. Lett., 2014, 5, 3345-3353.

35 K. Aryanpour, A. Shukla and S. Mazumdar, J. Phys. Chem. $C$, 2015, 119, 6966-6979.

36 D. Lehnherr, J. Gao, F. A. Hegmann and R. R. Tykwinski, Org. Lett., 2008, 10, 4779-4782.

37 D. Lehnherr, A. H. Murray, R. McDonald and R. R. Tykwinski, Angew. Chem., Int. Ed., 2010, 49, 61906194, (Angew. Chem., 2010, 122, 6326-6330).

38 D. Lehnherr and R. R. Tykwinski, Aust. J. Chem., 2011, 64, 919-929.

39 A. R. Waterloo, S. Kunakom, F. Hampel and R. R. Tykwinski, Macromol. Chem. Phys., 2012, 213, 10201032.

40 M. Gholami and R. R. Tykwinski, Chem. Rev., 2006, 106, 4997-5027.

41 P. A. Limacher and H. P. Luthi, Wiley Interdiscip. Rev.: Comput. Mol. Sci., 2011, 1, 477-486.

42 G. C. Solomon, D. Q. Andrews, R. H. Goldsmith, T. Hansen, M. R. Wasielewski, R. P. Van Duyne and M. A. Ratner, J. Am. Chem. Soc., 2008, 130, 17301-17308.

43 G. C. Solomon, D. Q. Andrews, R. P. Van Duyne and M. A. Ratner, J. Am. Chem. Soc., 2008, 130, 7788-7789.

44 G. C. Solomon, C. Herrmann, T. Hansen, V. Mujica and M. A. Ratner, Nat. Chem., 2010, 2, 223-228.

45 D. Q. Andrews, G. C. Solomon, R. P. Van Duyne and M. A. Ratner, J. Am. Chem. Soc., 2008, 130, 17309-17319.

46 C. Bosshard, R. Spreiter, P. Günter, R. R. Tykwinski, M. Schreiber and F. Diederich, Adv. Mater., 1996, 8, 231234.

47 D. Q. Andrews, G. C. Solomon, R. H. Goldsmith, T. Hansen, M. R. Wasielewski, R. P. V. Duyne and M. A. Ratner, J. Phys. Chem. C, 2008, 112, 16991-16998.

48 K. G. L. Pedersen, A. Borges, P. Hedegård, G. C. Solomon and M. Strange, J. Phys. Chem. C, 2015, 119, 26919-26924.

49 E. C. Greyson, J. Vura-Weis, J. Michl and M. A. Ratner, J. Phys. Chem. B, 2010, 114, 14168-14177.

50 P. M. Zimmerman, F. Bell, D. Casanova and M. HeadGordon, J. Am. Chem. Soc., 2011, 133, 19944-19952.
51 R. W. A. Havenith, H. D. de Gier and R. Broer, Mol. Phys., 2012, 110, 2445-2454.

52 P. E. Teichen and J. D. Eaves, J. Phys. Chem. B, 2012, 116, 11473-11481.

53 P. M. Zimmerman, C. B. Musgrave and M. Head-Gordon, Acc. Chem. Res., 2013, 34, 1339-1347.

54 N. Renaud, P. A. Sherratt and M. A. Ratner, J. Phys. Chem. Lett., 2013, 4, 1065-1069.

55 T. C. Berkelbach, M. S. Hybertsen and D. R. Reichman, J. Chem. Phys., 2013, 138, 114103.

56 D. Beljonne, H. Yamagata, J. L. Brédas, F. C. Spano and Y. Olivier, Phys. Rev. Lett., 2013, 110, 226402.

57 X. Feng, A. V. Luzanov and A. I. Krylov, J. Phys. Chem. Lett., 2013, 4, 3845-3852.

58 A. V. Akimov and O. V. Prezhdo, J. Am. Chem. Soc., 2014, 136, 1599-1608.

59 A. B. Kolomeisky, X. Feng and A. I. Krylov, J. Phys. Chem. C, 2014, 118, 5188-5195.

60 T. Zeng, R. Hoffmann and N. Ananth, J. Am. Chem. Soc., 2014, 136, 5755-5764.

61 D. Casanova, J. Chem. Theory Comput., 2014, 10, 324334.

62 A. J. Musser, M. Maiuri, D. Brida, G. Cerullo, R. H. Friend and J. Clark, J. Am. Chem. Soc., 2015, 137, 5130-5139.

63 T. Lange, J.-D. van Loon, R. R. Tykwinski, M. Schreiber and F. Diederich, Synthesis, 1996, 537-550.

64 A. R. Suárez and M. R. Mazzieri, J. Org. Chem., 1987, 52, 1145-1147.

65 P. S. Vincett, E. M. Voigt and K. E. Rieckhoff, J. Chem. Phys., 1971, 55, 4131-4140.

66 M. Maggini and D. M. Guldi, in Organic, Physical and Materials Photochemistry, ed. V. Ramamurthy and K. S. Schanze, Marcel Dekker, New York, 2000, vol. 6, pp. 149-196.

67 The rate constants for the diffusion controlled transduction of triplet excited state energy are $(2.4 \pm 0.1) \times 10^{9}$ and $(2.6 \pm$ $0.3) \times 10^{9} \mathrm{M}^{-1} \mathrm{~s}^{-1}$ for XC1 and XC2, respectively.

68 Details about 7 are reported in ref. 27.

69 D. M. Guldi and M. Prato, Acc. Chem. Res., 2000, 33, 695703.

70 N. V. Korovina, S. Das, Z. Nett, X. Feng, J. Joy, R. Haiges, A. I. Krylov, S. E. Bradforth and M. E. Thompson, J. Am. Chem. Soc., 2016, 138, 617-627.

71 J. J. Snellenburg, S. P. Laptenok, R. Seger, K. M. Mullen and I. H. M. van Stokkum, J. Stat. Soft., 2012, 49, 1-22.

72 Using the bleaching at around $625 \mathrm{~nm}$ to calculate the triplet quantum yields with the bleaching method, results in a similar trend. For XC1, the quantum yields are derived as $139 \pm 5 \%$ in toluene and $150 \pm 5 \%$ in benzonitrile, for XC2, the corresponding values are $139 \pm 5 \%$ and $180 \pm 5 \%$, respectively.

73 H. Nakamura and D. G. Truhlar, J. Chem. Phys., 2001, 115, 10353-10372.

74 H. Nakamura and D. G. Truhlar, J. Chem. Phys., 2002, 117, 5576-5593. 
75 A. Nitzan, Chemical Dynamics in Condensed Phases: Relaxation, Transfer, and Reactions in Condensed Molecular Systems, Oxford University Press, New York, 2014.

76 O. Treutler and R. Ahlrichs, J. Chem. Phys., 1995, 102, 346-354.

77 F. Weigend and R. Ahlrichs, Phys. Chem. Chem. Phys., 2005, 7, 3297-3305.

78 F. Weigend, Phys. Chem. Chem. Phys., 2006, 8, 1057-1065.

79 A. A. Granovsky, J. Chem. Phys., 2011, 134, 214113.

80 T. H. Dunning Jr. and P. J. Hay, in Methods of Electronic Structure Theory, ed. H. F. Schaefer III, Plenum Press, New York, 1997, vol. 3, pp. 1-28.

81 B. R. Brooks and H. F. Schaefer, J. Chem. Phys., 1979, 70, 5092-5106.

82 B. R. Brooks, W. D. Laidig, P. Saxe, N. C. Handy and H. F. Schaefer III, Phys. Scr., 1980, 21, 312-322.
83 H. A. Witek, Y.-K. Choe, J. P. Finley and K. Hirao, J. Comput. Chem., 2002, 23, 957-965.

84 M. W. Schmidt, K. K. Baldridge, J. A. Boatz, S. T. Elbert, M. S. Gordon, J. H. Jensen, S. Koseki, N. Matsunaga, K. A. Nguyen, S. Su, T. L. Windus, M. Dupuis and J. A. Montgomery, J. Comput. Chem., 1993, 14, 13471363.

85 TURBOMOLE V6.6 2014, a development of University of Karlsruhe and Forschungszentrum Karlsruhe $\mathrm{GmbH}$, 1989-2007, TURBOMOLE GmbH, since 2007, available from http://www.turbomole.com.

86 M. S. Gordon and M. W. Schmidt, in Theory and Applications of Computational Chemistry: the first forty years, ed. C. E. Dykstra, G. Frenking, K. S. Kim and G. E. Scuseria, Elsevier, Amsterdam, 2005, pp. 1167-1189. 